
Company
Products/activity
Location
URL

Protein preparation and mass spectrometry

Advion BioSciences Agilent

Amersham Biosciences

Applied Biosystems

ARRM

Beckman Coulter

Bio-Rad

Bruker Daltonics

Gyros

Hamilton Bonaduz

Intrinsic Bioprobes

KBiosystems

MDS Sciex

Micromass-Waters

Millipore

NextGen Sciences

Proteome Systems

Qiagen

RoboDesign

Shimadzu-Biotech

Thermo Finnigan

\section{Protein arrays}

Advalytix

Affibody

Affymetrix

BioGenex

HTS Biosystems

Molecular Probes

Molecular Staging

NanoPlex Technologies

Panomics

PerkinElmer Life Sciences

Phylos

Protometrix

Scienion

SomaLogic

Intellisense ESI chip for MS; contract liquid chromatography

Mass spectrometers; 1100 series systems for HPLC; capillary electrophoresis systems; workstation for RNA, DNA or protein analysis

Instruments, equipment and products for proteomics and cellular assays; Ettan range

of equipment for sample preparation and mass spectrometry

Protein sequencers, mass spectrometers, chromatography systems, peptide synthesizers;

ICAT stable-isotope labeling reagents

Robotic spot cutter and plate replicator

Automated tools for molecular biology, biochemistry, genomics and proteomics; ProteomeLab Fullerton, California

Products, instruments and software for life-sciences research; ProteomeWorks integrated

system; WorksBase bioinformatics software

Instruments, software and consumables for mass spectrometry for the life sciences;

Proteineer proteomics software suite

CD microlaboratory for protein preparation for mass spectrometry

Microlab workstations for liquid-handling, MALDI spotting and protein crystallization

Proteomics products and analysis based on proprietary BIA/MS technique

Automated equipment for proteomics

Mass spectrometer developers and producers

Micromass instruments for mass spectrometry; HPLC systems; reagents and consumables

Automated equipment for life sciences research; Montage In-Gel DigestZP Kit and

ZipPlate Micro-SPE plates for protein sample preparation from gel for MS

a2DE automated 2D-gel electrophoresis workstation

Proteomics technology; ProteomlQ integrated system for proteomics

BioRobot multifunctional laboratory workstation and equipment for automated nucleic acid, protein and cell purification; reagents, kits and instruments for proteomics

Automated equipment for biotechnology, protein structure analysis, genomics and proteomics

AXIMA mass spectrometers; XCISE integrated sample gel excision processor;

Chemical Inkjet Printer

Instruments for chromatography and mass spectrometry; ProteomeX workstation for

automated LC-MS protein analysis

ArrayBooster nanopump system for protein chip and microarray incubation

High-throughput protein identification and characterization by proprietary binding proteins

Spotters and arrayers

Automated DNA and protein chip processing and scanning

FLEX CHIP array platform for detection of protein binding using surface plasmon resonance

Fluorescent probes

Proprietary RCAT chip-based proteomics platform; whole-genome amplification kits Nanobarcodes metallic nanoparticle detection tags for proteomics and other applications TranSignal Human Cytokine Antibody Array for profiling expression of 18 human cytokines Automated systems for liquid handling and sample preparation; HydroGel BioChip; ProteinArray workstation for protein arrays

Trinectin binding proteins for protein capture

Yeast protein microarray for identification and interaction studies

sciCAPTURE customized protein array; sciLlSA antibody array

Photoaptamer arrays for proteomics

TeleChem/Arraylt International Protein Edition Spot Bot for making protein arrays

Zeptosens ZeptoMARK protein chip platform using planar optical waveguide technology

Zyomyx
Ithaca, New York

Palo Alto, California

Uppsala, Sweden

Foster City, California

Adelaide, Australia

Hercules, California

Billerica, Massachusetts

Uppsala, Sweden

Bonaduz, Switzerland

Tempe, Arizona

Basildon, UK

Ontario, Canada

Milford, Massachusetts

Bedford, Massachusetts

Huntingdon, UK

North Ryde, Australia

Venlo, the Netherlands

Carlsbad, California

Kyoto, Japan

San Jose, California

Munich, Germany

Stockholm, Sweden

Santa Clara, California

Ramon, California

Hopkinton, Massachusetts

Eugene, Oregon

New Haven, Connecticut

Mountain View, California

Redwood City, California

Boston, Massachusetts

Lexington, Massachusetts

Branford, Connecticut

Berlin, Germany

Boulder, Colorado

Sunnyvale, California

Witterswil, Switzerland

Hayward, California
wwW-advion.com

www.agilent.com

www5.amershambiosciences.com

home.appliedbiosystems.com

www.arrm.com

www.beckmancoulter.com

www.bio-rad.com

www.bdal.com

www.gyros.com

hamiltoncomp.com

www.intrinsicbio.com

www.kbiosystems.com

www.sciex.com

www.waters.com

www.millipore.com

www.nextgensciences.com

www.proteomesystems.com

www.qiagen.com

www.robodesign.com

www.shimadzu-biotech.net

www.thermo.com

www.advalytix.de

www.affibody.com

www.affymetrix.com

www.biogenex.com

www.htsbiosystems.com

www.probes.com

www.molecularstaging.com

www.nanoplextech.com

www.panomics.com

lifesciences.perkinelmer.com

www.phylos.com

www.protometrix.com

www.scienion.de

www.somalogic.com

www.arrayit.com

www.zeptosens.com

www.zyomyx.com

Protein crystallization and structure determination

BSI Proteomics

DataCentric Automation

Fluidigm

Gilson
Proprietary system for automated protein crystallization for crystallography

Automated systems for protein crystallography

Microfluidics chip for protein crystallization

Automated liquid-handling, pipetting and protein crystallization equipment
Gaithersburg, Maryland Nashville, Tennessee

South San Francisco, California

Middleton, Wisconsin www.bsiproteomics.com www.titanceg.com

www.fluidigm.com www.gilson.com 


$\begin{array}{ll}\begin{array}{l}\text { Company } \\ \text { Greiner Bio-One }\end{array} & \begin{array}{l}\text { Products/activity } \\ \text { Consumables for high-throughput research and diagnostics, microplates; Crystal Star } \\ \text { microplate plafforms for protein crystallization } \\ \text { Kpstate }\end{array} \\ \begin{array}{l}\text { KinaseProfiler specificity testing, IC5OProfiler Express and services for crystallography assay } \\ \text { development, bulk protein production and purification }\end{array} \\ \text { Inpharmatica } & \begin{array}{l}\text { Biopendium and CeleraEdition Biopendium proteome annotation resources; } \\ \text { PharmaCarta computational genomics system }\end{array}\end{array}$

\section{General}

20/20 GeneSystems

Applied Precision

Apogent Technologies

Antibodies by Design/

MorphoSys

Axon Instruments

Becton Dickinson/

BD Biosciences

Biacore

BioVisioN

BMG Labtechnologies

brainprot

Brinkmann

Chromagen

Complexbio

Cytomyx
Dynal
Eppendorf
Eurogentec
GeneProt

Genetix

Genomic Solutions

Hybrigenics

Invitrogen

Jerini Peptide Technology

Novagen

Peptide Specia

Procognia

Prodigene

Promega

Protagen

Proteome Sciences

Roche Diagnostics

Schleicher \& Schuell

Stratagene

Tecan

Trenzyme

Xzillion
P-FILM Layered Gene Scanning membrane system for protein or nucleic acid capture arrayWoRxe biochip reader

Labware and equipment for the life sciences

Recombinant antibody library for custom generation of monoclonal antibodies

GenePix 4000B microarray scanner and GenePix Pro microarray analysis software Culture media, radioassay kits, FACS range of flow cytometers; monoclonal antibodies, antibody arrays for proteomics; reagents and biochemicals for molecular biology Analysis and measurement of biomolecular interactions using surface plasmon resonance Peptidomics: technologies for discovery and identification of peptides and small proteins Microplate and array readers

Proteomics analysis

Laboratory instrument suppliers; software; consumables

Fluorescence based products, kits and services for gene expression and protein assay Proteome analysis of biological membra microdomains, and protein interactions

1 - and $2 \mathrm{D}$ gel analysis, mass spectrometry and amino-terminal sequencing for proteins

Dynabeads for immunoprecipitation and phage-display panning

Liquid-handling equipment, centrifuges

Large-format 2DE; protein identification by MALDI, ESI/MS/MS, Edman sequencing Industrial-scale separation, identification and characterization of human proteins

Picking, arraying, gridding and replicating robots for genomics and proteomics Investigator automated system for proteomics; software, consumables and services In silico prevalidation, and experimental validation of drug targets

Kits and reagents for proteomics, molecular and cell biology

High-density peptide microarrays; kinase identification and substrate profiling

Reagents for genomics and proteomics

Custom peptides

Glycoanalysis of glycoproteins

Protein production in plants; vaccine development

Vectors, reagents and kits for genomics, proteomics and cellular analysis

Protein analysis services

Drug target and disease biomarker discovery; ProteoSHOP toolkit for high-output proteomics

Reagents and kits for molecular biology, functional genomics and proteomics research

Slides, kits, equipment and reagents for protein microarrays

Tools and reagents for proteomics

Automated solutions for proteomics: ProTeam free-flow electrophoresis for fractionation, automated 2D-PAGE spot picking, in-gel digester and interface to mass spectrometer Genomics and proteomics services: cloning, mutagenesis, library construction, gene expression, protein purification

High-throughput protein profiling using protein sequence tag technology
Location

Frickenhausen, Germany

URL

Charlottesville, Virginia

London, UK

(n)

www.upstate.com

www.inpharmatica.com
Rockville, Maryland

Issaquah, Washington

Portsmouth, New Hampshire

Martinsried, Germany

Foster City, California

Franklin Lakes, New Jersey

Uppsala, Sweden

Hannover, Germany

Offenburg, Germany

Vienna, Austria

Westbury, New York

San Diego, California

March, Germany

Cambridge, UK

Oslo, Norway

Hamburg, Germany

Seraing, Belgium

Geneva, Switzerland

New Milton, UK

Ann Arbor, Michigan

Paris, France

Carlsbad, California

Berlin, Germany

Madison, Wisconsin

Heidelberg, Germany

Maidenhead, UK

College Station, Texas

Madison, Wisconsin

Dortmund, Germany

Cobham, UK

Lewes, UK

Keene, New Hampshire

La Jolla, California

Männedorf, Switzerland

Konstanz, Germany

Frankfurt, Germany

www.2020gene.com

www.appliedprecision.com

www.apogent.com

www.antibodyservices.com

www.axon.com

www.bd.com

www.biacore.com

www.biovision-discovery.de

www.bmg-labtechnologies.com

gert.lubec@akh-wien.ac.at

www.brinkmann.com

www.chromagen.com

www.complexio-gmbh.com

www.cytomyx.com

www.dynalbiotech.com

www.eppendorf.com

www.eurogentec.com

www.geneprot.com

www.genetix.co.uk

www.genomicsolutions.com

www.hybrigenics.com

www.invitrogen.com

www.jerini.com

www.novagen.com

www.peptide.de

www.procognia.com

www.prodigene.com

www.promega.com

www.protagen.com

www.proteome.co.uk

www.roche-applied-science.com

www.schleicher-schuell.

com/bioscience

www.stratagene.com

www.tecan.com

www.trenzyme.com

www.xzillion.com

San Diego, California Kortrijk, Belgium

Newtown, Connecticut

Tel-Aviv, Israel

Greifswald, Germany

Geneva, Switzerland

Palo Alto, California

Bethesda, Maryland

Heidelberg, Germany

London, UK

Wyndmoor, Pennsylvania

Newcastle-upon-Tyne, UK

St Marcel, France

Oslo, Nonway

San Diego, California www.accelrys.com

www.applied-maths.com

www.arraygenetics.com

www.cgen.com

www.decodon.de

www.genebio.com

www.incyte.com

www.informaxinc.com

www.lionbioscience.com

www.matrixscience.com

www.megametrics.com

www.nonlinear.com

www.proteomicsolutions.fr

www.pubgene.com

www.strubix.com

PubGene

Structural Bioinformatics

PubGene databases and analysis software for genomics and proteomics

Protein and protein-structure databases; computational proteomics for drug discovery 